\title{
The homogeneity and heterogeneity of occurrence, characteristics, and prognosis in hepatocellular carcinoma patients with synchronous and metachronous bone metastasis
}

\author{
Yanting Zhang ${ }^{1,2^{*}}$, Yao $\mathrm{Xu}^{1,2^{*}}$, Wenjuan $\mathrm{Ma}^{1,2^{*}}$, Haixiao $\mathrm{Wu}^{1,2}$, Guijun $\mathrm{Xu}^{2,3}$, Vladimir P. Chekhonin ${ }^{2,4}$, Karl \\ Peltzer ${ }^{2,5}$, Xin Wang ${ }^{2,6}$, Guowen Wang ${ }^{1 凶}$, Chao Zhang ${ }^{1,2 \bowtie}$ \\ 1. Tianjin Medical University Cancer Institute and Hospital, National Clinical Research Center for Cancer, Key Laboratory of Cancer Prevention and Therapy, \\ Tianjin's Clinical Research Center for Cancer, Tianjin, China. \\ 2. The Sino-Russian Joint Research Center for Bone Metastasis in Malignant Tumor, Tianjin, China. \\ 3. Department of Orthopedics, Tianjin Hospital, Tianjin University, Tianjin, China. \\ 4. Department of Basic and Applied Neurobiology, Federal Medical Research Center for Psychiatry and Narcology, Moscow, Russian Federation. \\ 5. Department of Psychology, University of the Free State, Turfloop, South Africa. \\ 6. Department of Epidemiology and Biostatistics, West China School of Public Health, Sichuan University, South Renmin Road, Wuhou District, Chengdu, \\ China. \\ *These authors contributed equally to this work.
}

$\triangle$ Corresponding authors: Guowen Wang, Department of Bone and Soft Tissue Tumors, Tianjin Medical University Cancer Institute and Hospital, Tianjin, China. Tel: +86 18622221109; E-mail:wangguowen@tmu.edu.cn; Chao Zhang, Department of Bone and Soft Tissue Tumors, Tianjin Medical University Cancer Institute and Hospital, Tianjin, China. Tel: +86 15222093833; E-mail: drzhangchao@tmu.edu.cn.

(1) The author(s). This is an open access article distributed under the terms of the Creative Commons Attribution License (https://creativecommons.org/licenses/by/4.0/). See http://ivyspring.com/terms for full terms and conditions.

Received: 2021.07.24; Accepted: 2021.11.22; Published: 2022.01.01

\begin{abstract}
Purpose: Based on the one of the largest hepatocellular carcinoma $(\mathrm{HCC})$ population with bone metastasis (BM) from the single center in Tianjin, China, the present study aimed to investigate the risk and survival of synchronous bone metastasis (sBM) and metachronous bone metastasis (mBM) in HCC, and to reveal characteristics and related factors of $\mathrm{HCC}$ patients with bone metastasis.

Methods: HCC patients with bone metastasis between 2009 and 2017 from Tianjin Medical University Cancer Institute \& Hospital, Tianjin, China, were involved. Chi-square test/ Fisher's exact test and Logistic regression were used to estimate the risk factors of bone metastasis in HCC. Kaplan-Meier method was used to estimate the survival of HCC patients, and the Log-rank test was used to analyze the survival of HCC patients. The prognostic factors of HCC patients with BM were identified via Kaplan-Meier method and multivariable COX regression model.

Results: Among $4421 \mathrm{HCC}$ patients, 128 patients with BM were identified. Of the 128 patients with BM, 77 patients $(60.16 \%)$ were with sBM and 51 patients (39.84\%) were with $\mathrm{mBM}$. The incidence of $\mathrm{sBM}$ in $\mathrm{HCC}$ was $1.74 \%$ at initial diagnosis. The most common metastatic site of sBM was rib, followed by lumbar, thoracic, and sacral. The median latency time from HCC diagnosis to $\mathrm{mBM}$ was six months. The most common site of $\mathrm{mBM}$ was thoracic, followed by lumbar, sacral and rib. Alcohol-drinking history $(P=0.027)$, numbers $(P=0.023)$ and size $(P=0.008)$ of intrahepatic tumor, lymph node metastasis $(P<0.001)$, serum ALP $(P=0.004)$ and HGB $(P=0.004)$ level were found to be correlated with the occurrence of $B M$. The overall survival between non-BM and $B M$ were statistically different $(P=0.028)$.

Conclusion: The incidence of sBM in HCC was $1.74 \%$ at initial diagnosis. The median latency time from HCC diagnosis to $\mathrm{mBM}$ was 6 months. The characteristics between occurrence and prognosis showed significant difference between sBM and mBM. Early identification of high-risk BM population was essential for the improvement of both quality of life and prognosis. The revealed related factors can potentially guide $s B M$ and $\mathrm{mBM}$ identification and early diagnosis in HCC.
\end{abstract}

Key words: hepatocellular carcinoma, synchronous bone metastasis, metachronous bone metastasis, risk, prognosis 


\section{Introduction}

Hepatocellular carcinoma (HCC) was the fifth common cancer in the world, and it was the second most fatal tumor [1, 2]. It was globally estimated that 250,000 people died of HCC each year. Most HCC patients were diagnosed at the advanced stage, thus HCC patients usually showed the poor prognosis. The 5-year survival rate of HCC patients was reported to be less than $20 \%[3,4]$. Bone metastasis (BM) was accepted as one of the factors that impeded both the survival and quality of life for HCC patients. A total of $25 \%$ HCC patients showed BM at initial diagnosis or at their later course. BM rarely resulted in the death of HCC patients, but the pain and dysfunction caused by BM significantly reduced the patients' quality of life. To improve the quality of life and the survival of patients, the early diagnosis of BM in HCC is important.

The most metastatic sites in HCC patients with $\mathrm{BM}$ were reported to be spine, pelvis, and ribs, of which spinal metastasis accounts for $40 \%[5,6]$. Skeletal related events (SREs), resulted by BM, were defined as severe pain, pathological fractures, malignant hypercalcemia, spinal cord compression, and other neurological compression syndrome [7]. SREs dramatically increased the analgesic use and reduced the quality of patients' life. As reported in the previous studies, once BM occurred in HCC patients, the 2-year survival was less than 5\% [8]. According to the occurrence time of $\mathrm{BM}, \mathrm{BM}$ is divided into synchronous BM (sBM) and metachronous BM (mBM). SBM was defined as the occurrence of BM at HCC diagnosis, while $\mathrm{mBM}$ was defined as the occurrence of $\mathrm{BM}$ at patients' later course. Considering different diagnosis and treatment procedures, clinicopathological characteristics and prognostic outcomes of sBM and mBM in HCC can be found. However, constricted by the sample size, seldom study reported the difference between sBM and $\mathrm{mBM}$ in HCC. In HCC patients with sBM, the proper diagnosis of $\mathrm{sBM}$ can guide the prognostic prediction and the individualized treatment generation. While in the patients with $\mathrm{mBM}$, timely screening of $\mathrm{BM}$ is of significance on guiding bone targeting therapy. Thus, the study looking into sBM and $\mathrm{mBM}$ in HCC is warrant.

Based on a single center population, we systematically evaluated the difference on occurrence, progression and prognosis between $\mathrm{sBM}$ and $\mathrm{mBM}$ patients with HCC. The results in the present study can potentially guide individualized $\mathrm{BM}$ screening and treatment for BM in HCC.

\section{Patients and methods}

Patients who were diagnosed with HCC between January 2009 and December 2017 in Tianjin Medical University Cancer Institute \& Hospital (Tianjin, China), were retrospectively identified. The inclusion criteria were as follows: 1 . over the age of 18; 2. diagnosed as BM by histopathological and/or imaging examinations such as standard X-rays, whole-body bone scans, computed tomography (CT), magnetic resonance imaging (MRI) and positron emission tomography-computed tomography (PET$\mathrm{CT})$; 3. without other malignant tumors or severe organic disease; 4 . with definitive information of the bone metastatic site(s). HCC patients with non-BM were randomly drawn from the same period. In order to compare the characteristics of patients with bone metastasis and those without bone metastasis, we performed $1: 2$ population matching to determine the sample size of non-BM group. In our study, mBM was defined when HCC was diagnosed prior to the BM diagnosis more than 3 months, otherwise patients were considered to be the part of the sBM (Figure 1). The overall survival (OS) was defined as the time from HCC diagnosis to cancer-associated death or to the end of follow up. The time of follow-up was performed from HCC diagnosis to death or January 2020. Latency time in HCC patients with $\mathrm{mBM}$ referred to the time from HCC diagnosis to $\mathrm{BM}$ occurrence, and survival after BM was defined as the time from BM diagnosis to death or the end of follow-up. This study was approved by the Ethics Committee of Tianjin Medical University Cancer Institute and Hospital, Tianjin, China.

Patient demographics and tumor variables were collected. Patient demographic variables, including gender, age at diagnosis, $\mathrm{ABO}$ blood type and related medical history. A series of tumor characteristics were collected, including number, size, serum ALP and AFP, blood cell counts, and metastatic site (bone, lymph node and other sites), as well as treatment modalities, such as surgical type. Information on both the number and specific sites of BM in HCC were collected.

\section{Statistical analyses}

All statistical analyses were performed using SPSS version 21.0 (IBM, Armonk, NY). We used method of multiple imputation to handle missing data. The Chi-square test or Fisher's exact test was used to make the consistent of the baseline characteristics of HCC patients with/without BM. Variables that had an association with a $\mathrm{P}<0.2$ based on univariate analysis were included in multivariate logistic regression model. OS was assessed with the Kaplan-Meier method, and the Log-rank test was 
used to study subgroups. Those variables with $P$ value $<0.2$ in the Kaplan-Meier analysis were included in the multivariate COX regression analysis. Factors in which $P<0.05$ based on COX regression analyses were identified as the independent prognostic variables. And we used Cox regression analysis to calculate the hazard ratio (HR) and 95\% confidence intervals $(95 \% \mathrm{CI})$. It was considered statistically significant if $P<0.05$.

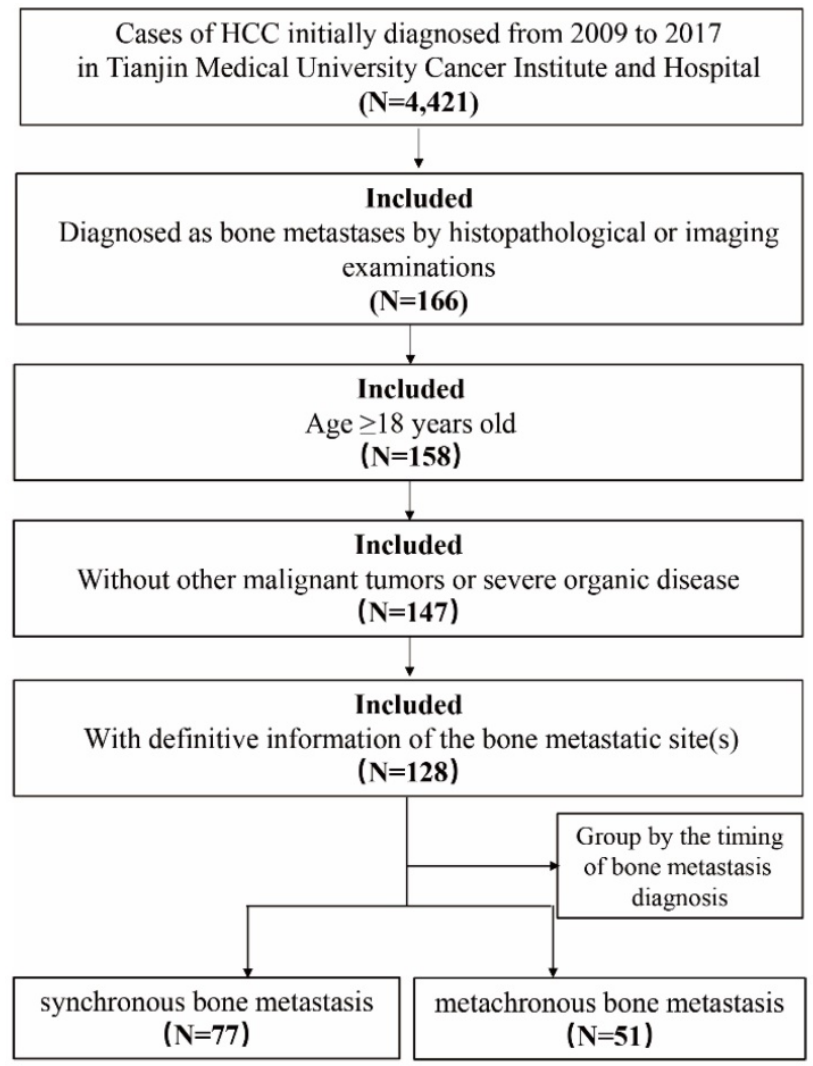

Figure 1. Flowchart of the patient selection process in the present study

\section{Results}

\section{Characteristics of study population}

HCC patients who were diagnosed with HCC between January 2009 and December 2017 in Tianjin Medical University Cancer Institute and Hospital (Tianjin, China), were retrospectively identified. A total of 4421 patients were diagnosed as HCC in our hospital, including 166 patients with BM. According to the inclusion and exclusion criteria, a total of 128 patients were included. There were 77 patients with sBM, including 66 male patients $(85.7 \%), 11$ female patients $(14.3 \%)$, and aged $\leq 40,41-60$, and $\geq 61$ years old patients were $3(3.9 \%), 49$ (63.6\%), 25 (32.5\%) respectively; There were 51 patients with $\mathrm{mBM}, 48$ male patients $(94.1 \%), 3$ female patients $(5.9 \%)$, and 3 (5.9\%), $28(54.9 \%)$, and $20(39.2 \%)$ patients with age $\leq 40,41-60$, and $\geq 61$, respectively. The median age of patients was 57 years (range 49-63) in sBM group and 56 years (range 51-64) in mBM group respectively. In addition, 263 HCC patients with non-BM were randomly selected. The population distribution of HCC patients was shown in Table 1.

Table 1. The population distribution of HCC patients with BM and non-BM

\begin{tabular}{llllll}
\hline Variable & Overall, & Non-BM, & BM, & sBM, & mBM, \\
& $\mathrm{N}=391$ & $\mathrm{~N}=263$ & $\mathrm{~N}=128$ & $\mathrm{~N}=77$ & $\mathrm{~N}=51$ \\
\hline Male gender & $339 / 391$ & $225 / 263$ & $114 / 128$ & $66 / 77$ & $48 / 51$ \\
Age at diagnosis, $\leq 60$ years & $251 / 391$ & $168 / 263$ & $83 / 128$ & $52 / 77$ & $31 / 51$ \\
ABO blood type & & & & & \\
A & $101 / 385$ & $71 / 263$ & $30 / 122$ & $18 / 76$ & $12 / 46$ \\
B & $106 / 385$ & $77 / 263$ & $29 / 122$ & $24 / 76$ & $5 / 46$ \\
AB & $65 / 385$ & $38 / 263$ & $27 / 122$ & $9 / 76$ & $18 / 46$ \\
O & $113 / 385$ & $77 / 263$ & $36 / 122$ & $25 / 76$ & $11 / 46$ \\
Smoking history & $161 / 391$ & $107 / 263$ & $54 / 128$ & $38 / 77$ & $16 / 51$ \\
Alcohol-drinking history & $110 / 391$ & $81 / 263$ & $29 / 128$ & $18 / 77$ & $11 / 51$ \\
Family history of cancer & $75 / 391$ & $52 / 263$ & $23 / 128$ & $11 / 77$ & $12 / 51$ \\
Antiviral history & $54 / 391$ & $41 / 263$ & $13 / 128$ & $5 / 77$ & $8 / 51$ \\
Intrahepatic tumor numbers, single & $225 / 385$ & $170 / 263$ & $55 / 122$ & $33 / 76$ & $22 / 46$ \\
Intrahepatic tumor size, $\leq 5 \mathrm{~cm}$ & $203 / 362$ & $154 / 244$ & $49 / 118$ & $30 / 72$ & $19 / 46$ \\
Vascular tumor thrombus & $132 / 385$ & $98 / 263$ & $34 / 122$ & $21 / 76$ & $13 / 46$ \\
Intrahepatic metastasis & $30 / 391$ & $20 / 263$ & $10 / 128$ & $6 / 77$ & $4 / 51$ \\
Extraosseous metastases & $53 / 391$ & $26 / 263$ & $27 / 128$ & $15 / 77$ & $12 / 51$ \\
Lymph node metastasis & $53 / 391$ & $19 / 263$ & $34 / 128$ & $24 / 77$ & $10 / 51$ \\
AFP, $\geq 25$ ng/ml & $216 / 385$ & $140 / 263$ & $76 / 122$ & $46 / 76$ & $30 / 46$ \\
ALP, $>150$ U/L & $135 / 376$ & $74 / 260$ & $61 / 116$ & $42 / 74$ & $19 / 42$ \\
HBsAg, positive & $289 / 385$ & $200 / 263$ & $89 / 122$ & $54 / 76$ & $35 / 46$ \\
HGB, (g/L) & & & & & \\
high (>160) & $54 / 380$ & $44 / 259$ & $10 / 121$ & $4 / 75$ & $6 / 46$ \\
low (<110) & $24 / 380$ & $7 / 259$ & $17 / 121$ & $10 / 75$ & $7 / 46$ \\
normal (110-160) & $302 / 380$ & $208 / 259$ & $94 / 121$ & $61 / 75$ & $33 / 46$ \\
WBC, (109/L) & & & & & \\
high $(>10)$ & $30 / 380$ & $13 / 259$ & $17 / 121$ & $13 / 75$ & $4 / 46$ \\
low $(<4)$ & $69 / 380$ & $49 / 259$ & $20 / 121$ & $9 / 75$ & $11 / 46$ \\
normal (4 10) & $282 / 380$ & $197 / 259$ & $85 / 121$ & $53 / 75$ & $32 / 46$ \\
PLT, (10 $/ \mathrm{L})$ & & & & & \\
high (>300) & $32 / 380$ & $16 / 259$ & $16 / 121$ & $9 / 75$ & $7 / 46$ \\
low (<100) & $60 / 380$ & $38 / 259$ & $22 / 121$ & $11 / 75$ & $11 / 46$ \\
normal (100 300) & $289 / 380$ & $205 / 259$ & $84 / 121$ & $55 / 75$ & $29 / 46$ \\
\hline
\end{tabular}

\section{Metastatic sites}

In 128 HCC patients with BM, a total of 297 metastatic sites of bone were found. A total of 201 sites were found in sBM while 96 sites were found in $\mathrm{mBM}$. The most common metastatic site in sBM was ribs (41 sites), followed by lumbar (34 sites), thoracic (31 sites), and sacral (24 sites). The most common metastatic site in mBM was thoracic (23 sites), followed by lumbar ( 21 sites), sacral (15 sites) and ribs (10 sites). Together, the most common metastatic site was trunk (231sites, $77.8 \%$ ), followed by upper limb (38 sites, $12.8 \%$ ), lower limb (16 sites, $5.4 \%$ ), and skull (12 sites, $4.0 \%$ ).

In HCC patients with $\mathrm{mBM}$, the most common bone metastatic site at 6,12 , and 24 months after HCC diagnosis was the trunk, with the incidences of $52.1 \%$, $62.5 \%$, and $72.9 \%$, respectively (Figure $2 \mathrm{~A}$ ). After Log-Rank test, the difference between trunk and non-trunk in the incidence of BM was statistically 
significant $(P<0.001)$. Among all metastatic sites, the most common metastatic site was the thoracic. The risk of metastasis at 6,12 , and 24 months after HCC diagnosis was $69.6 \%, 82.6 \%$, and $87.0 \%$, respectively (Figure 2B). At 6, 12 and 24 months after HCC diagnosis, the commonly found bone metastatic sites were thoracic, thoracic, and sacral, respectively. Among the 128 HCC patients with BM, 101 patients were found to be with bone-only metastases, and 27 patients were found to be with multi organs metastasis. The most common metastasis site was lung $(53.0 \%)$, while the least was brain (2.9\%) (Supplementary Table S1).

\section{Risk factors of BM in HCC}

As shown in Table 2, univariate and multivariate analyses of risk factors for HCC patients with BM showed that there was no significant difference in gender, age, $\mathrm{ABO}$ blood type, smoking history, family history of cancer, antiviral history, vascular tumor thrombus, intrahepatic metastasis, extraosseous metastases, serum AFP, HBsAg, WBC and PLT level between $\mathrm{BM}$ patients and non-BM patients. There were significant differences in alcohol-drinking history, numbers and size of intrahepatic tumor, lymph node metastasis, serum ALP and HGB level. Multivariate analysis showed that HGB level $<110$ $\mathrm{g} / \mathrm{L} \quad(P=0.004, \mathrm{OR}=5.026)$, serum ALP $>150 \mathrm{U} / \mathrm{L}$ $(P=0.004, \quad \mathrm{OR}=2.271)$, presence of lymph node metastasis $(P<0.001, \mathrm{OR}=4.073)$ were correlated with BM occurrence in HCC. Solitary intrahepatic tumor $(P=0.008, \mathrm{OR}=0.491)$, intrahepatic tumor size $\leq 5 \mathrm{~cm}$ $(P=0.023, \mathrm{OR}=0.530)$ and presence of alcohol-drinking history were protective factors for BM occurrence in HCC. Figure 3 showed forest plot of multivariate analysis for risk factors associated with BM occurrence.
A

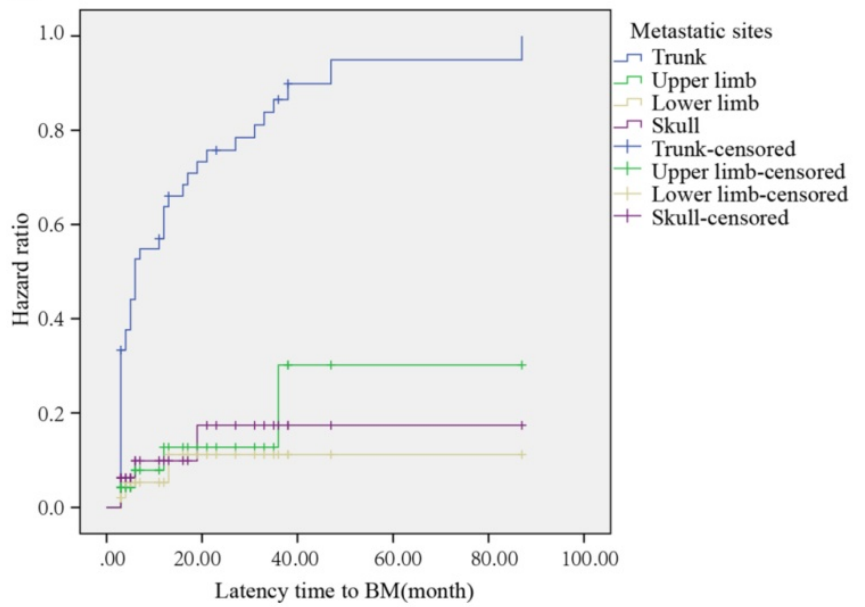

B

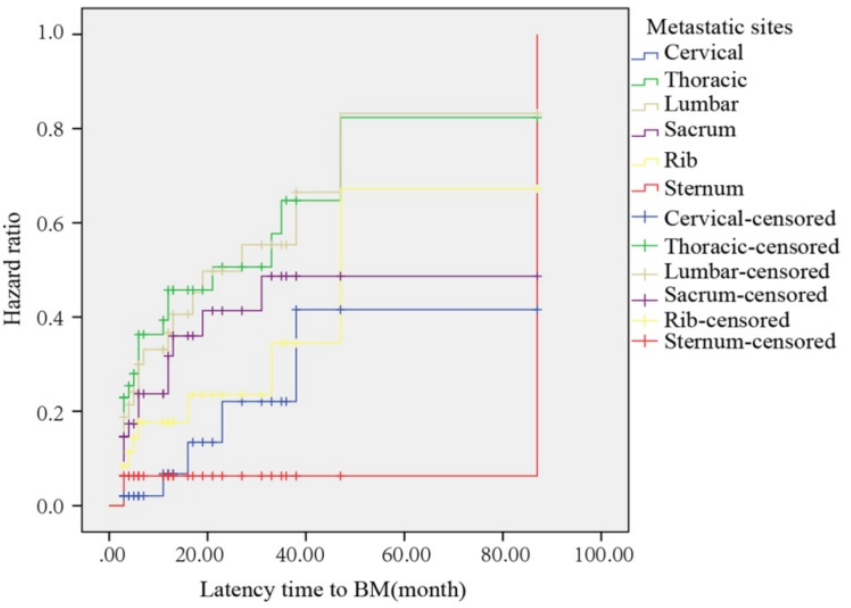

Figure 2. (A) Hazard curve of whole body bone metastases after HCC diagnosis. (B) Hazard curve of trunk metastases after HCC diagnosis.

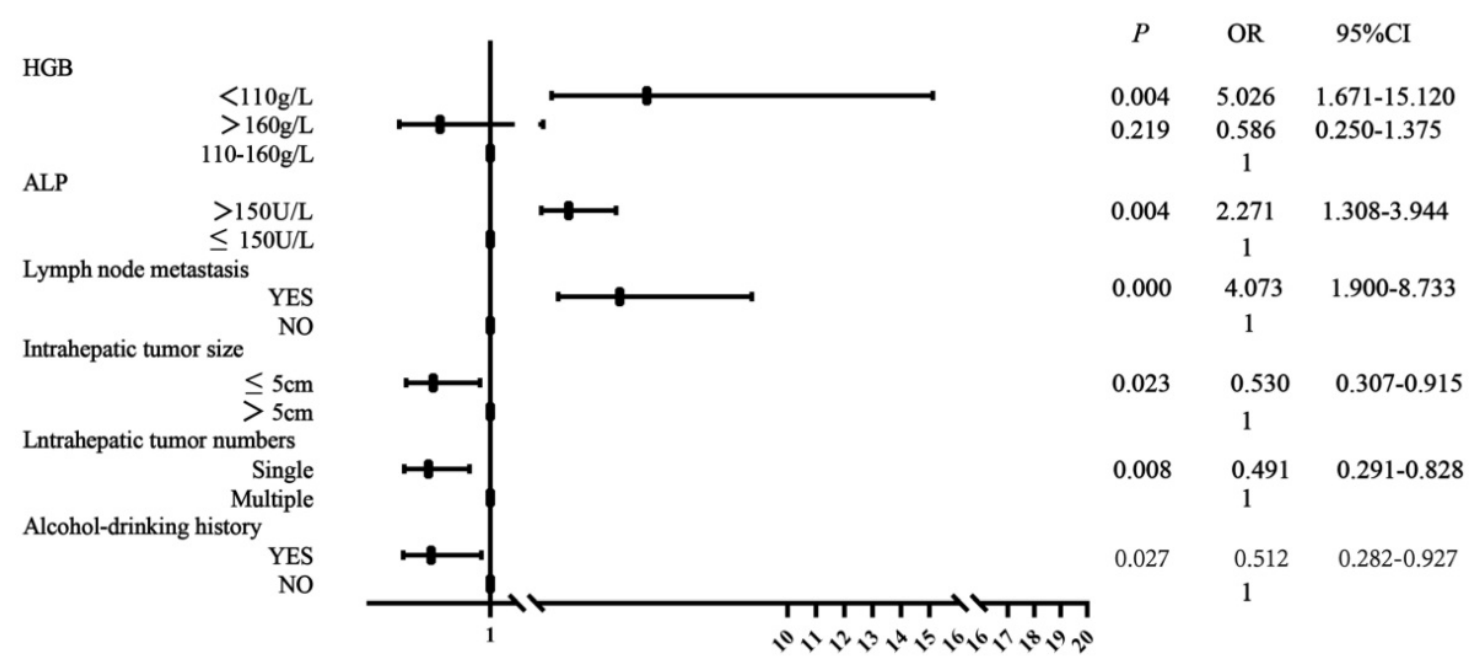

Figure 3. Forest plot of risk factors for BM occurrence. OR (odds ratio), $95 \% \mathrm{Cl}$ (confidence interval). 
Table 2. The baseline characteristics comparison of HCC patients with BM and non-BM and risk factor analysis of BM

\begin{tabular}{|c|c|c|c|c|c|}
\hline \multirow[t]{2}{*}{ Variable } & \multirow{2}{*}{$\begin{array}{l}\text { Chi-Squared Test } \\
P\end{array}$} & \multicolumn{2}{|c|}{ Univariate analysis } & \multicolumn{2}{|c|}{ Multivariate analysis } \\
\hline & & $P$ & OR & $P$ & OR $(95 \% \mathrm{CI})$ \\
\hline Male gender & 0.337 & 0.339 & 1.375 & & \\
\hline Age at diagnosis, $\leq 60$ years & 0.852 & 0.852 & 1.043 & & \\
\hline ABO blood type & 0.199 & & & & \\
\hline A & & 0.733 & 0.904 & & \\
\hline B & & 0.467 & 0.806 & & \\
\hline $\mathrm{AB}$ & & 0.195 & 1.520 & & \\
\hline $\mathrm{O}$ & & & 1(reference) & & \\
\hline Smoking history & 0.777 & 0.777 & 1.064 & & \\
\hline Alcohol-drinking history & 0.093 & 0.094 & 0.658 & 0.027 & $0.512(0.282-0.927)$ \\
\hline Family history of cancer & 0.671 & 0.671 & 0.889 & & \\
\hline Antiviral history & 0.144 & 0.147 & 0.612 & & \\
\hline Intrahepatic tumor numbers, single & $<0.001$ & $<0.001$ & 0.449 & 0.008 & $0.491(0.291-0.828)$ \\
\hline Intrahepatic tumor size, $\leq 5 \mathrm{~cm}$ & 0.001 & $<0.001$ & 0.415 & 0.023 & $0.530(0.307-0.915)$ \\
\hline Vascular tumor thrombus & 0.001 & 0.072 & 0.651 & & \\
\hline Intrahepatic metastasis & 0.942 & 0.942 & 1.030 & & \\
\hline Extraosseous metastases & 0.002 & 0.003 & 2.437 & & \\
\hline Lymph node metastasis & $<0.001$ & $<0.001$ & 4.645 & $<0.001$ & $4.073(1.900-8.733)$ \\
\hline $\mathrm{AFP}, \geq 25 \mathrm{ng} / \mathrm{ml}$ & 0.01 & 0.096 & 1.452 & & \\
\hline $\mathrm{ALP},>150 \mathrm{U} / \mathrm{L}$ & $<0.001$ & $<0.001$ & 2.788 & 0.004 & $2.271(1.308-3.944)$ \\
\hline HBsAg, positive & 0.002 & 0.514 & 0.850 & & \\
\hline $\operatorname{HGB}(\mathrm{g} / \mathrm{L})$ & $<0.001$ & & & & \\
\hline high $(>160)$ & & 0.064 & 0.503 & 0.219 & $0.586(0.250-1.375)$ \\
\hline low $(<110)$ & & $<0.001$ & 5.374 & 0.004 & $5.026(1.671-15.120)$ \\
\hline normal (110-160) & & & 1(reference) & & 1(reference) \\
\hline $\operatorname{WBC}\left(10^{9} / \mathrm{L}\right)$ & 0.006 & & & & \\
\hline high $(>10)$ & & 0.005 & 3.031 & & \\
\hline low $(<4)$ & & 0.851 & 0.946 & & \\
\hline normal $(4 \sim 10)$ & & & 1(reference) & & \\
\hline $\operatorname{PLT}\left(10^{9} / \mathrm{L}\right)$ & 0.019 & & & & \\
\hline high $(>300)$ & & 0.018 & 2.440 & & \\
\hline low $(<100)$ & & 0.245 & 1.413 & & \\
\hline normal (100 300) & & & 1(reference) & & \\
\hline
\end{tabular}

Table 3. Multivariate analysis for sBM compared with non-BM

\begin{tabular}{llllll}
\hline Variable & \multicolumn{2}{l}{$\begin{array}{l}\text { Univariate } \\
\text { analysis }\end{array}$} & \multicolumn{3}{l}{ Multivariate analysis } \\
\cline { 2 - 6 } & $P$ & OR & $P$ & OR & $95 \%$ CI \\
\hline Intrahepatic tumor size, $\leq 5 \mathrm{~cm}$ & 0.001 & 0.417 & 0.031 & 0.486 & $0.252-0.936$ \\
lymph node metastasis & $<0.001$ & 5.815 & 0.001 & 4.332 & $1.831-10.249$ \\
$\begin{array}{l}\text { ALP }>150 \text { U } / \mathrm{L} \\
\text { HGB }(\mathrm{g} / \mathrm{L})\end{array}$ & $<0.001$ & 3.299 & 0.003 & 2.666 & $1.391-5.111$ \\
low $(<110)$ & 0.002 & 4.871 & 0.040 & 3.909 & $1.067-14.324$ \\
\hline
\end{tabular}

Table 4. Multivariate analysis for mBM compared with non-BM

\begin{tabular}{llllll}
\hline Variable & \multicolumn{3}{l}{$\begin{array}{l}\text { Univariate } \\
\text { analysis }\end{array}$} & \multicolumn{3}{l}{ Multivariate analysis } \\
\cline { 2 - 6 } & $P$ & OR & $P$ & OR & $95 \%$ CI \\
\hline Male gender & 0.109 & 2.702 & 0.012 & 12.132 & $1.744-84.401$ \\
ABO blood type & & & & & \\
AB & 0.005 & 3.316 & 0.024 & 3.239 & $1.167-8.989$ \\
Alcohol-drinking history & 0.188 & 0.618 & 0.027 & 0.354 & $0.142-0.886$ \\
Intrahepatic tumor numberds,single & 0.032 & 0.501 & 0.046 & 0.449 & $0.204-0.987$ \\
Extraosseous metastases & 0.008 & 2.805 & 0.016 & 3.769 & $1.284-11.060$ \\
HGB (g/L) & & & & & \\
low $(<110)$ & 0.001 & 6.303 & $<0.001$ & 17.627 & $3.787-82.041$ \\
PLT (10/L) & & & & & \\
low $(<100)$ & 0.070 & 2.046 & 0.039 & 2.893 & $1.053-7.949$ \\
\hline
\end{tabular}

After univariate and multivariate analyses, intrahepatic tumor size, lymph node metastasis, serum ALP and HGB levels were related with sBM occurrence in HCC. Gender, ABO blood type, alcoholdrinking history, intrahepatic tumor numbers, extraosseous metastases, HGB and PLT level were relevant to $\mathrm{mBM}$ occurrence. The related factors of HCC patients with sBM and mBM were illustrated in Table 3 and Table 4, respectively. Patients with lymph node metastasis, serum ALP $>150 \mathrm{U} / \mathrm{L}$, and HGB $<110 \mathrm{~g} / \mathrm{L}$ were more likely to occur sBM compared with non-BM, while intrahepatic tumor size $\leq 5 \mathrm{~cm}$ was the protective factor for sBM occurrence. Compared with non-BM, male gender, $\mathrm{AB}$ blood type, presence of extraosseous metastases, HGB $<110 \mathrm{~g} / \mathrm{L}$ and PLT $<100 \times 10^{9} / \mathrm{L}$ were risk factors for $\mathrm{mBM}$. Patients with solitary intrahepatic tumor and alcoholdrinking history were less likely to occur mBM. Forest plots of relative factors associated with $\mathrm{sBM}$ and $\mathrm{mBM}$ occurrence were illustrated in Supplementary Figure S1 and Supplementary Figure S2.

\section{Survival estimation in HCC patients with BM}

To the end of the follow-up, a total of 83 patients completed whole follow-up (40 non-BM cases/43 BM cases). The median survival of HCC patients in the non-BM and BM groups were 16.07 months and 8.61 months, respectively. Among $43 \mathrm{BM}$ patients with complete follow-up information (21 mBM cases/22 sBM cases), the median survival of patients in the sBM and $\mathrm{mBM}$ groups were 6.51 months and 11.73 months, respectively. After Log-rank test, the differences of overall survival between non-BM and BM $(P=0.028)$, 
sBM and $\mathrm{mBM}(P=0.026)$ were statistically significant (Figure 4). For patients with $\mathrm{mBM}$, the distribution of spinal metastasis after HCC diagnosis was illustrated in Supplementary Table S2, and the median latency time to BM was six months.

The median survival after BM was 4.27 months in HCC patients with mBM while 6.97 months in HCC patients with sBM. And after Log-Rank test, there was no statistical difference on survival after $\mathrm{BM}$ diagnosis between patients with $\mathrm{mBM} / \mathrm{sBM}(P=0.344)$ (Figure 5).

\section{Prognostic analysis}

Kaplan-Meier analysis and Cox regression model were used to identify prognostic factors for overall survival in HCC patients with BM. Besides the

A

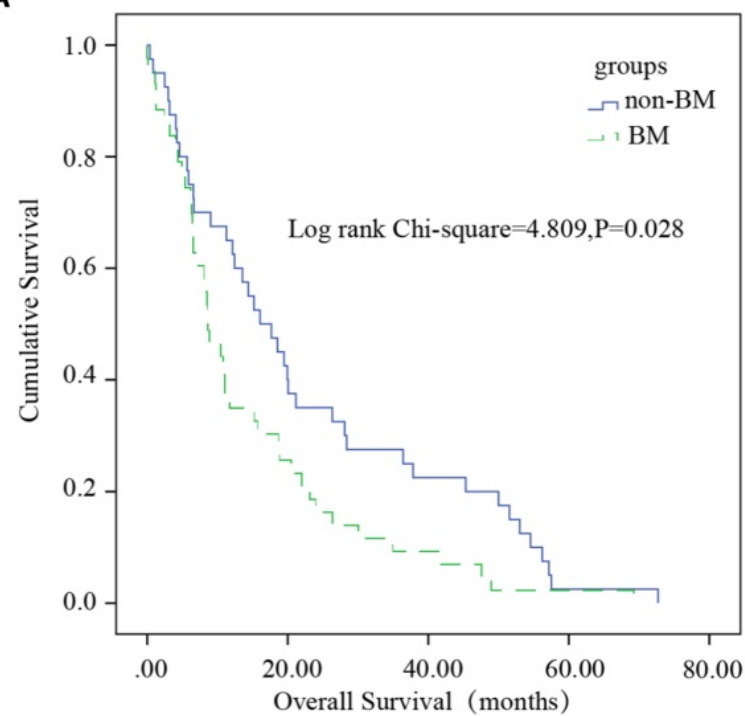

aforementioned involved variables, bone involvement numbers and bisphosphonates were included. A total of twenty-three variables were applied for univariate analysis, $P$-values less than 0.2 were included in the multivariable analysis. After univariate analysis, eight variables including intrahepatic tumor size, vascular tumor thrombus, serum ALP, HGB, WBC, PLT, TACE and bone involvement numbers were included for Cox regression analysis. Through overall survival analysis, vascular tumor thrombus was shown to be independent prognostic factor for BM patients with HR $3.114(P=0.026)$, as shown in Table 5. Compared with the patients without vascular tumor thrombus, the patients with vascular tumor thrombus suggested the worse prognosis (Figure S3).

B

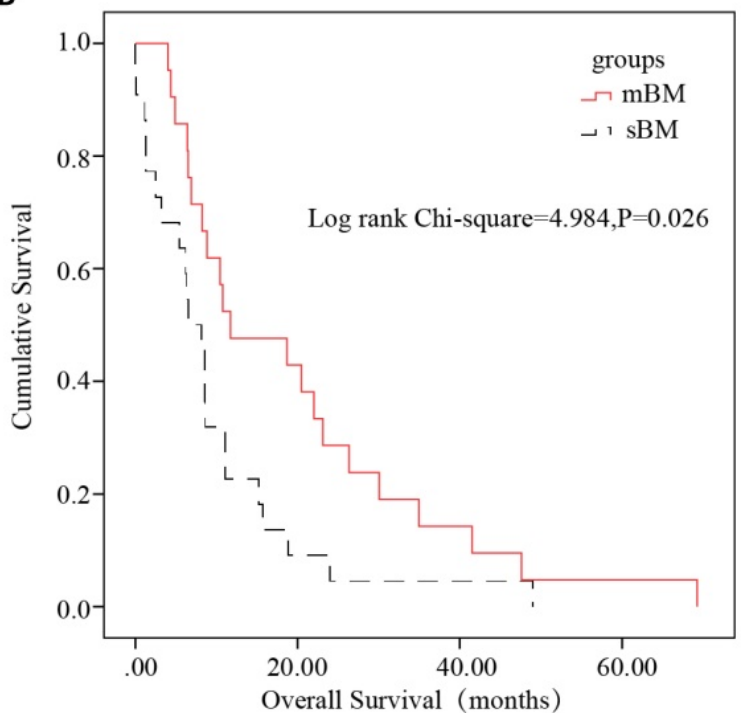

Figure 4. Kaplan-Meier overall survival analysis of HCC patients. $P<0.05$ was considered to be statistically significant. (A) Survival analysis was performed between non-BM and BM. The survival of $\mathrm{HCC}$ patients with $\mathrm{BM}$ was significantly worse $(P=0.028)$. (B) Survival analysis was performed between $\mathrm{sBM}$ and $\mathrm{mBM}$. The survival of $\mathrm{HCC}$ patients with sBM was significantly worse than $\mathrm{mBM}(P=0.026)$.

Table 5. Univariate and multivariate analyses of prognostic factors in BM groups

\begin{tabular}{|c|c|c|c|c|c|c|c|}
\hline \multirow[t]{2}{*}{ Variable } & \multirow[t]{2}{*}{ Non-BM, $\mathrm{N}=40$} & \multirow[t]{2}{*}{$\mathrm{BM}, \mathrm{N}=43$} & \multicolumn{2}{|c|}{ Univariate analysis } & \multicolumn{2}{|c|}{ Multivariable analysis } & \multirow[b]{2}{*}{$95 \% \mathrm{CI}$} \\
\hline & & & $\chi^{2}$ & $P$ & $P$ & HR & \\
\hline Intrahepatic tumor size, $\leq 5 \mathrm{~cm}$ & $19 / 37$ & $16 / 41$ & 4.213 & 0.122 & 0.778 & 0.896 & $0.417-1.925$ \\
\hline Vascular tumor thrombus & $17 / 40$ & $13 / 42$ & 4.775 & 0.092 & 0.026 & 3.114 & $1.144-8.475$ \\
\hline ALP $>150 \mathrm{U} / \mathrm{L}$ & $18 / 40$ & $23 / 40$ & 7.126 & 0.028 & 0.167 & 2.077 & $0.737-5.849$ \\
\hline HBsAg, positive & $28 / 40$ & $29 / 42$ & 2.800 & 0.247 & & & \\
\hline \multicolumn{8}{|l|}{$\operatorname{HGB}(g / L)$} \\
\hline high $(>160)$ & $5 / 40$ & $2 / 41$ & 2.882 & 0.090 & 0.770 & 0.697 & $0.062-7.824$ \\
\hline low $(<110)$ & $2 / 40$ & $7 / 41$ & 0.087 & 0.768 & 0.400 & 1.536 & $0.565-4.176$ \\
\hline normal (110 160) & $33 / 40$ & $32 / 41$ & 0.799 & 0.371 & & 1(reference) & \\
\hline \multicolumn{8}{|l|}{ WBC $\left(10^{9} / \mathrm{L}\right)$} \\
\hline high $(>10)$ & $4 / 40$ & $6 / 41$ & 2.377 & 0.123 & 0.205 & 0.420 & $0.110-1.605$ \\
\hline low $(<4)$ & $6 / 40$ & $7 / 41$ & 4.173 & 0.030 & 0.587 & 1.527 & $0.331-7.057$ \\
\hline normal $(4 \sim 10)$ & $30 / 40$ & $28 / 41$ & 7.043 & 0.008 & & 1(reference) & \\
\hline \multicolumn{8}{|l|}{ PLT $\left(10^{9} / \mathrm{L}\right)$} \\
\hline high $(>300)$ & $6 / 40$ & $6 / 41$ & 4.474 & 0.034 & 0.136 & 0.436 & $0.147-1.298$ \\
\hline low $(<100)$ & $6 / 40$ & $10 / 41$ & 5.328 & 0.021 & 0.488 & 0.613 & $0.153-2.446$ \\
\hline normal (100 300) & $28 / 40$ & $25 / 41$ & 5.106 & 0.024 & & 1(reference) & \\
\hline Primary tumor surgery & $26 / 40$ & $7 / 41$ & $<0.001$ & 0.990 & & & \\
\hline TACE & $7 / 40$ & $5 / 43$ & 1.941 & 0.164 & 0.412 & 1.573 & $0.533-4.642$ \\
\hline Bone involvement, solitary & $16 / 42$ & $16 / 42$ & 4.832 & 0.089 & 0.375 & 0.674 & $0.282-1.611$ \\
\hline
\end{tabular}




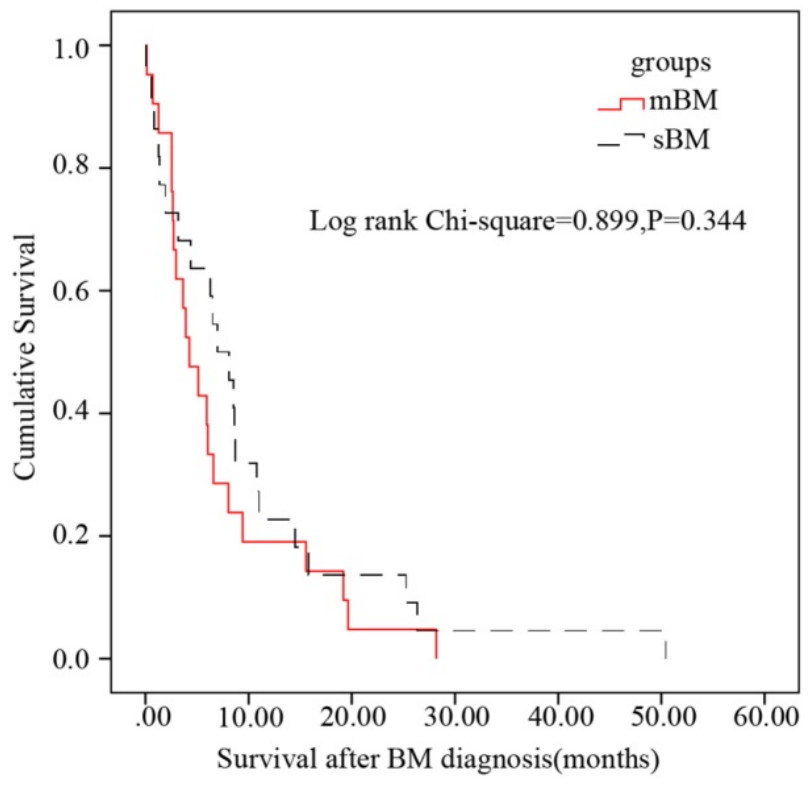

Figure 5. Kaplan-Meier survival after BM analysis in subsets of patients with BM. No statistical difference between patients with $\mathrm{mBM}$ and patients with $\mathrm{sBM}$ was found $(\mathrm{P}=0.344)$.

\section{Discussion}

With the development of diagnosis and treatment technology, the survival of HCC patients has been improved. The incidence of BM in HCC has been increasing [9]. The incidence of BM in HCC was reported to increase from $4.5 \%$ during $1978-1987$ to 12.9\% during 1988-1997 [9].

The process of BM in HCC was accepted as a multi-step process, including cancer cells detach from the primary site, blood vessels invasion, distal capillaries migration and attachment into the bone, extravasate, blood supply recruitment and adjacent tissues invasion [10].

Different managements were given to HCC patients with sBM and mBM. Since BM was a significant restraint on the survival of HCC patients, the exclusion of sBM should be performed once HCC was diagnosed. Prognostic estimation should be firstly performed, and bone target therapy should be then considered once sBM was diagnosed in HCC patients [11]. Currently, there has been no widely accepted BM screening strategy in HCC, early BM diagnosis can significantly improve the quality of life. Thus, the prediction on metastatic site and BM occurrence time in HCC patients with $\mathrm{mBM}$ are warrant. The present study gave the reference on both the specific metastatic site and time of $\mathrm{mBM}$ occurrence in HCC.

Patients with BM were usually with poor prognosis. Early detection of BM is crucial for patients to receive timely treatment. Therefore, the identification of high-BM risk is important. Due to the rarity of skeletal involvement from HCC, few studies looking into risk factors for BM in HCC was previously performed. Some studies reported that the expression of connective tissue growth factor (CTGF), interleukin-11 (IL-11) [12], chemokine receptor CXCR4 [13] and LncRNA34a [14] can be potentially valuable predictive biomarkers for BM in HCC. Based on the Surveillance, Epidemiology, and End Results (SEER) database, it was shown that gender, marital status, T stage, lymph node involvement, intrahepatic metastases, and extrahepatic metastases were predictors of BM in HCC [5]. In this study, we found that serum HGB and ALP level, and presence of lymph node metastasis were significantly correlated with BM occurrence in HCC. Tumor size was reported to be positively correlated with distant metastasis in HCC, tumor size ( $>58 \mathrm{~mm}$ ) was 5.7 times more likely to develop distant metastasis than that of tumor less than $30 \mathrm{~mm}$, and 2.9 times than that of tumor size $30-58 \mathrm{~mm}$ [15].The grade malignancy of the primary tumor can be significantly affected by $\mathrm{T}$ grade. This can partially explain the potential mechanism of our results. As previously reported in breast cancer, the larger size of the primary tumor found, the higher risk of the metastasis developed [16]. Decreased HGB level was found to be one of the risk factors for sBM. Previous study reported that the decreased HGB level was associated with the metastatic risk in prostate cancer, which was consistent with our results [17]. The mechanism underlying HGB and sBM occurrence needs to be further studied.

Previous studies suggested that the most common BM site in HCC was the axial bone, which may be caused by the formation of portal hypertension and collateral networks throughout the vertebral vein system [18]. In our study, the most common BM site was the trunk bone. A total of 231 bone metastatic sites were found, including the lumbar vertebrae (55 sites) and thoracic vertebrae (54 sites), followed by the ribs (51 sites). For the first time, based on the single center of HCC cohort, we summarized the site-time data on mBM in HCC. Our results can potentially guide the clinical management and BM screening in HCC.

$\mathrm{BM}$ was a poor prognostic factor of patients with $\mathrm{HCC}$, and the overall survival of patients with BM is worse than that of patients without BM. And the prognosis of $\mathrm{BM}$ patients can be even worse when the patients be diagnosed with vascular tumor thrombus. Previous studies showed that factors associated with the prognosis of HCC patients with BM were radiotherapy, chemotherapy and lung metastasis [19]. Oral sorafenib in patients with extrahepatic metastasis can significantly improve the survival [20].

Since the study was a retrospective study, there 
were some limitations in the present study. Child-pugh classification, KPS score, and the degree of pathological grade of HCC were not collected in our dataset. Being lack of the external cohort, we were not able to perform external validation. In addition, incomplete follow-up data due to patient contact changes or poor adherence can affect the reliability of the data to some extent and we will also further expand the sample size to reduce bias.

\section{Conclusion}

The incidence of sBM in HCC was $1.74 \%$ at initial diagnosis. The median latency time from HCC diagnosis to mBM was six months. Among HCC patients, lymph node metastasis, serum ALP $>150$ $\mathrm{U} / \mathrm{L}$, and HGB < $110 \mathrm{~g} / \mathrm{L}$ were correlated with sBM occurrence. Male gender, $\mathrm{AB}$ blood type, presence of extraosseous metastases, HGB $<110 \mathrm{~g} / \mathrm{L}$ and PLT $<100 \times 10^{9} / \mathrm{L}$ were associated with $\mathrm{mBM}$ occurrence. Among HCC patients with BM, the most common metastatic site was trunk, followed by upper limb bone, lower limb and skull. Such factors and characteristics can be potentially used for individualized BM screening and early diagnosis. A poor prognosis (median survival 8.61 months) was reported in our study. HCC patients with $\mathrm{mBM}$ showed better median survival than the patients with sBM. Vascular thrombosis was found to be correlated with the significant worse prognosis.

\section{Abbreviations}

HCC: hepatocellular carcinoma; BM: bone metastasis; sBM: synchronous bone metastasis; mBM: metachronous bone metastasis; ALP: alkaline phosphatase; HGB: hemoglobin; PLT: platelet; WBC: white blood cell; TACE: Transarterial chemoembolization; AFP: alphafetoprotein.

\section{Supplementary Material}

Supplementary figures and tables. https://www.jcancer.org/v13p0393s1.pdf

\section{Acknowledgements}

\section{Funding}

The present study was sponsored by the National Natural Science Foundation of China (82011530050, 81801781, 82072004, 81903398).

\section{Author Contributions}

YTZ, YX and WJM were responsible for experimental design, experimental analysis and thesis writing. HXW, GJX, VP.C, K.P and XW participated in editing the manuscript. GWW and CZ were responsible for the guidance and review of the thesis.
All authors contributed to the article and approved the submitted version.

\section{Competing Interests}

The authors have declared that no competing interest exists.

\section{References}

1. Erkan B, Meier J, Clark T, Kaplan J, Lambert J, Chang S. Non-invasive diagnostic criteria of hepatocellular carcinoma: Comparison of diagnostic accuracy of updated LI-RADS with clinical practice guidelines of OPTN-UNOS, AASLD, NCCN, EASL-EORTC, and KLSCG-NCC. PloS one. 2019; 14: e0226291.

2. Jemal A, Ward E, Johnson C, Cronin K, Ma J, Ryerson B, et al. Annual Report to the Nation on the Status of Cancer, 1975-2014, Featuring Survival. Journal of the National Cancer Institute. 2017; 109(9):djx030.

3. Clark H, Carson W, Kavanagh P, Ho C, Shen P, Zagoria R. Staging and current treatment of hepatocellular carcinoma. Radiographics : a review publication of the Radiological Society of North America, Inc. 2005: S3-23.

4. Lin S, Hoffmann K, Schemmer P. Treatment of hepatocellular carcinoma: a systematic review. Liver cancer. 2012; 1: 144-58.

5. Guo X, Xu Y, Wang X, Lin F, Wu H, Duan J, et al. Advanced Hepatocellular Carcinoma with Bone Metastases: Prevalence, Associated Factors, and Survival Estimation. Medical science monitor : international medical journal of experimental and clinical research. 2019; 25: 1105-12.

6. Lu Y, Hu J, Lin X, Li X. Bone metastases from hepatocellular carcinoma: clinical features and prognostic factors. Hepatobiliary \& pancreatic diseases international: HBPD INT. 2017; 16: 499-505.

7. Roodman G. Mechanisms of bone metastasis. The New England journal of medicine. 2004; 350: 1655-64.

8. Seong J, Koom W, Park H. Radiotherapy for painful bone metastases from hepatocellular carcinoma. Liver international : official journal of the International Association for the Study of the Liver. 2005; 25: 261-5.

9. Fukutomi M, Yokota M, Chuman H, Harada H, Zaitsu Y, Funakoshi A, et al. Increased incidence of bone metastases in hepatocellular carcinoma. European journal of gastroenterology \& hepatology. 2001; 13: 1083-8.

10. Zheng Y, Zhou H, Dunstan C, Sutherland R, Seibel M. The role of the bone microenvironment in skeletal metastasis. Journal of bone oncology. 2013; 2: 47-57.

11. Coleman R, Croucher P, Padhani A, Clézardin P, Chow E, Fallon M, et al. Bone metastases. Nature reviews Disease primers. 2020; 6: 83

12. Xiang $Z$, Zeng $Z$, Tang $Z$, Fan J, He J, Zeng H, et al. Potential prognostic biomarkers for bone metastasis from hepatocellular carcinoma. The oncologist. 2011; 16: 1028-39.

13. Xiang Z, Zeng Z, Tang Z, Fan J, Zhuang P, Liang Y, et al. Chemokine receptor CXCR4 expression in hepatocellular carcinoma patients increases the risk of bone metastases and poor survival. BMC cancer. 2009; 9: 176.

14. Zhang L, Niu H, Ma J, Yuan B, Chen Y, Zhuang Y, et al. The molecular mechanism of LncRNA34a-mediated regulation of bone metastasis in hepatocellular carcinoma. Molecular cancer. 2019; 18: 120.

15. Yan B, Bai D, Zhang C, Qian J, Jin S, Jiang G. Characteristics and risk differences of different tumor sizes on distant metastases of hepatocellular carcinoma: A retrospective cohort study in the SEER database. International journal of surgery (London, England). 2020; 80: 94-100.

16. Sopik V, Narod S. The relationship between tumour size, nodal status and distant metastases: on the origins of breast cancer. Breast cancer research and treatment. 2018; 170: 647-56.

17. Cotogno P, Ranasinghe L, Ledet E, Lewis B, Sartor O. Laboratory-Based Biomarkers and Liver Metastases in Metastatic Castration-Resistant Prostate Cancer. The oncologist. 2018; 23: 791-7.

18. Longo V, Brunetti O, D'Oronzo S, Ostuni C, Gatti P, Silvestris F. Bone metastases in hepatocellular carcinoma: an emerging issue. Cancer metastasis reviews. 2014; 33: 333-42.

19. Hu C, Yang J, Huang Z, Liu C, Lin Y, Tong Y, et al. Diagnostic and prognostic nomograms for bone metastasis in hepatocellular carcinoma. BMC cancer. 2020; 20: 494.

20. Cheng A, Kang Y, Chen Z, Tsao C, Qin S, Kim J, et al. Efficacy and safety of sorafenib in patients in the Asia-Pacific region with advanced hepatocellular carcinoma: a phase III randomised, double-blind, placebo-controlled trial. The Lancet Oncology. 2009; 10: 25-34. 